\title{
AutoJoin: Providing Freedom from Specifying Joins
}

\author{
Terrence Mason \\ Iowa Database and Emerging Applications Laboratory, Computer Science \\ University of Iowa \\ Email: terrence-mason, lixin-wang,ramon-lawrence@uiowa.uiowa.edu \\ Lixin Wang \\ Iowa Database and Emerging Applications Laboratory \\ University of Iowa \\ Ramon Lawrence \\ Iowa Database and Emerging Applications Laboratory, Computer Science \\ University of Iowa
}

Keywords: Inference, Query, Interface, Schema, Ambiguity, Database.

\begin{abstract}
SQL is not appropriate for casual users as it requires understanding relational schemas and how to construct joins. Many new query interfaces insulate users from the logical structure of the database, but they require the automatic discovery of valid joins. Although specific query interfaces implement join determination algorithms, they are tied to the specific language and typically limited in scope or scalability. AutoJoin provides a general solution to the query inference problem, which allows more complex queries to be executed on larger and more complicated schemas. It enumerates query interpretations at least an order of magnitude faster than previous methods. In addition, the engine reduces the number of queries considered ambiguous. Experimental results demonstrate that query inference can be efficiently performed on large, complex schemas allowing simpler access to databases through keyword search or conceptual query languages. AutoJoin also provides programmers with a tool to iteratively create SQL queries without requiring explicit knowledge of the structure of a database.
\end{abstract}

\section{INTRODUCTION}

Despite significant improvement in the performance of database systems, the usability of databases has not improved at a similar pace. Although more usable query interfaces, including conceptual, graphical, and keyword languages, have been developed, they require the automatic determination of joins to complete the queries. The lack of a consistent join determination approach, which scales to large schemas, continues to limit the capabilities of new query interfaces. Even worse, the size and complexity of database schemas continues to grow, especially as global schemas are constructed for integrated systems. Users need a simpler method for querying larger and more complicated databases.

One of the major challenges in generating SQL queries is using joins to connect concepts or attributes located in different tables. Constructing these joins in SQL or through a graphical query interface is tedious, error-prone, and not intuitive to casual users (Catarci, 2000). Recent research using keyword searches on relational databases (Hristidis and Papakonstantinou, 2002; Balmin et al., 2004; Agrawal et al., 2002) allows the extraction of data without any required knowledge about the schema or metadata. These interfaces match keywords either to the data or the metadata of a database, which then requires the determination of the joins to relate the relations containing the keywords.

Our goal is to produce a query inference engine that dynamically converts an attribute-only or keyword query to SQL. For example, consider the equivalent keyword, conceptual and SQL queries on the TPC- $\mathrm{H}^{1}$ schema shown in Figure 1. It is desirable for the query system to automatically infer the SQL query from the simpler keyword and conceptual queries. This allows the creation of SQL queries without requiring explicit knowledge of the database.

The challenge is to make query inference effi cient, general, and practical for use in production databases. The query inference system should be query language independent to not restrict join determination to a particular query language. It also is critical that the overhead of query inference be minimal even for very large schemas.

Although ambiguity cannot be eliminated by a query inference strategy (since it is inherent in the relational schema), approaches to recognize and deal

\footnotetext{
${ }^{1}$ http://www.tpc.org/tpch/
} 


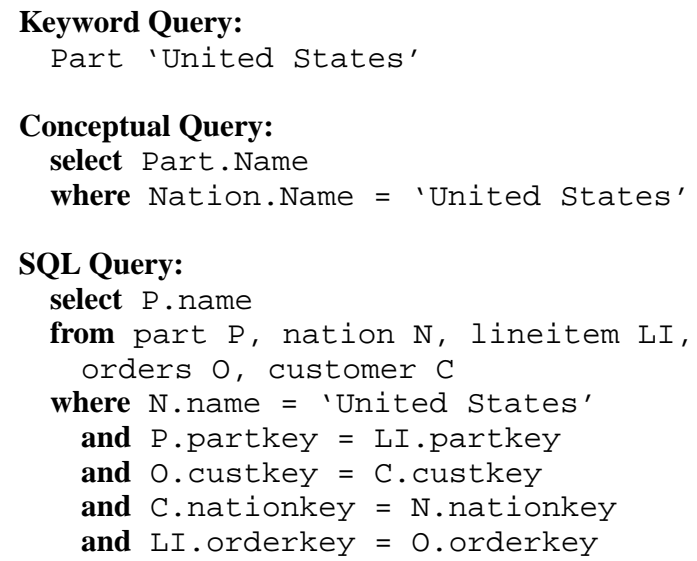

Figure 1: Equivalent Keyword, Conceptual, and Inferred SQL Queries on TPC-H

with ambiguity are necessary to make query inference valuable. Two specifi $\mathrm{c}$ forms of ambiguity that may lead to multiple query interpretations are addressed. In addition, schemas may have multiple sets of joins that are equivalent in their semantic meaning. By identifying and reducing these duplicate join paths to a single core path, ambiguity is reduced.

The AutoJoin inference engine effi ciently extends the capabilities of previous inference approaches, while maintaining independence from particular query languages or interfaces. The individual contributions of AutoJoin are:

- An algorithm called EMO that effi ciently constructs all maximal sets of lossless joins in a schema. EMO signifi cantly outperforms previous approaches that fail on large schemas.

- A method for reducing the number of ambiguous queries by detecting and removing semantically equivalent interpretations.

- Effi cient algorithms for generating query interpretations at query execution time.

- An extension of the lossless join approach to generate queries with a lossy join.

- A performance study that demonstrates the approach is scalable.

The rest of this paper is organized as follows. Section 2 provides background on query inference strategies. Section 3 presents the AutoJoin inference engine along with the join graph structure. Section 4 describes the overall strategy for precomputing the lossless join trees by the EMO algorithm. Effi cient join determination algorithms are presented in Section 5. A performance study in Section 6 shows that query inference can be performed with minimal overhead even for large schemas. The paper then closes with future work and conclusions.

\section{Background}

State of the art database interfaces require query inference, as users should not be required to know the schema and structure of the database queried. Keyword searches along with natural language querying require an effi cient, scalable, and general strategy to discover joins for query execution . An ideal query inference engine would automatically apply to existing relational schemas without administrator intervention, quickly pre-compute the necessary data structures to minimize overhead during query execution, and return a ranked list of query interpretations based on specifi cations from the query interface.

The Universal Relation provided the fi rst interface which required query inference. The lossless join property related to functional dependencies provided the mechanism to determine the joins required to complete the query (Maier and Ullman, 1983). If more than one lossless interpretation exists, the inferred query results in the union of all the unique lossless queries. In another approach, the query with the lowest cost (Wald and Sorenson, 1984) is selected as the inferred query. The cost function fi rst identifi es a lossless interpretation. If lossless joins do not exist for the query, lossy joins are permitted. These two approaches both infer a single query, while new interfaces require a ranked list of interpretations.

The pursuit of simpler interfaces for relational databases has led to varying methods of join determination. Each keyword search interface has developed their own algorithm to address the challenges of fi nding the k-lowest cost query interpretations for a set of keywords. Discover (Hristidis and Papakonstantinou, 2002) grows all ways from a relation containing one of the keywords with a limit on the number of joins permitted between relations. This ineffi ciently generates extra graphs that do not contain all the keywords. DBXplorer (Agrawal et al., 2002) identifi es the location of keywords through an effi cient symbol table and then infers the joins required for query interpretations by generating spanning trees. Neither approach maintains the lossless property in their cost functions and all computations occur at query time.

Conceptual query languages aim to hide the complexity of the schema from users by mapping concepts familiar to the user to the relational model. Again, these languages require the identifi cation of joins to complete the query. CQL (Owei and Navathe, 2001) uses a shortest path algorithm to fi nd the minimum join paths between specifi ed concepts resulting in a single query interpretation. Another conceptual 
model (Zhang et al., 1999) requires an administrator to semi-automatically generate and annotate a semantic graph of the database and then uses a search algorithm constrained by limiting both the number of joins and interpretations generated. Natural language interfaces (Popescu et al., 2003) map a natural language query to concepts represented in the database, then the joins required to connect all concepts must be determined.

Obviously, query inference is critical to the development of advanced user interfaces. However, each inference system directly links their join determination algorithms to a specifi c query interface with a hard-coded mapping algorithm to generate the SQL query. These solutions are inherently not confi gurable. The goal is to isolate query inference at the schema level away from the query interface, to focus on the overall challenges of query inference. By solving the query inference problem in general, the AutoJoin inference engine will allow the development of query interfaces without the constraint of a specific join determination algorithm.

\section{Architecture}

The AutoJoin architecture consists of a preprocessing step to identify maximal lossless joins and a query-time inference engine to determine joins for the query interface. In the pre-processing step, schema information is extracted from relational databases using standard API calls and stored in a generic schema representation called a join graph. The attributes and relations are optionally annotated to improve names and reduce ambiguity. Next, the system computes the maximal lossless joins and stores all information into an XML document for future QueryBuilder loads.

The query interface (see Figure 2) may be any text language or graphical query tool. The query interface allows the user to enter queries and then translates the user's query into a set of nodes and edges on the join graph. Nodes represent relations and edges represent joins. The query interface may provide a cost function, mapping costs to edges and nodes to rank the interpretations. The QueryBuilder takes this information and uses the pre-computed information to enumerate the potential interpretations. The Generator constructs all interpretations and passes them to the Ranker which uses any supplied ranking function to order the interpretations. Finally, the query interface uses the Iterator to return the interpretations in rank order. The query interface then executes the queries on the database. Since the architecture only requires the query interface to specify the nodes of interest, the query interface has complete

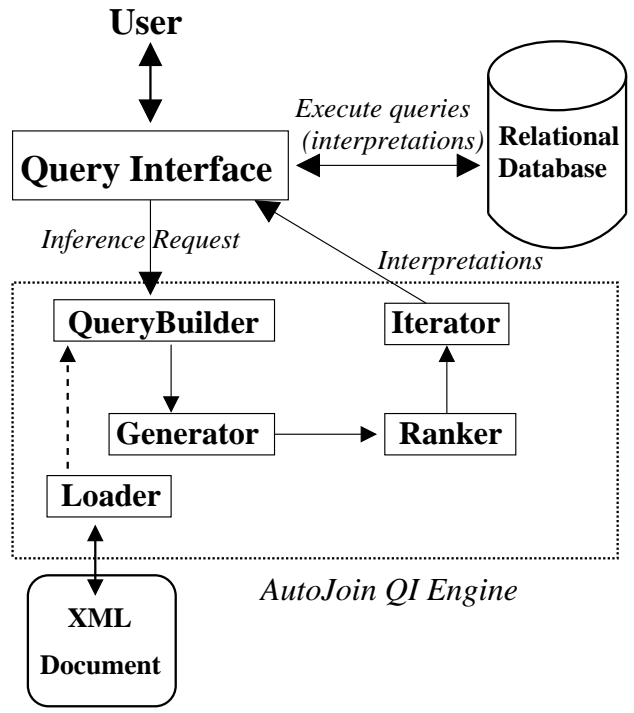

Figure 2: AutoJoin Architecture

control over the inference process. The key components of the architecture are discussed in the following sections.

\subsection{Representing Joins of a Schema}

The potential joins in a relational schema are represented by a directed graph called a join graph.

Definition 1 A join graph $J G=(N, E)$ for relational database schema $S$ is a directed graph where:

- Each relational schema $R_{i} \in S$ is represented as a node $n_{i} \in N$.

- There is a directed edge $e=\left(n_{i}, n_{j}\right) \in E$ from node $n_{i}$ (relation $R_{i}$ ) to node $n_{j}$ (relation $R_{j}$ ) if there exists a foreign key constraint of the form $R_{i}[A] \subseteq R_{j}[B]$ where $A$ and $B$ are subsets of the attributes of $R_{i}$ and $R_{j}$ respectively.

A join graph can be automatically built from an existing relational schema by extracting relation names and foreign key constraints. The example TPC-H schema used throughout this paper is in Figure 3, and its associated join graph is in Figure 4. Joins may be added to the join graph to identify additional potential joins. It is possible to have multiple edges between two nodes if there are two or more foreign keys between them. Determining the maximal set of lossless joins reduces to the problem of fi nding all connected, maximal subtrees of the join graph, as the lossless property is captured in the direction of the edges.

We now formally defi ne the meaning of a query, query interpretation, and query inference. 


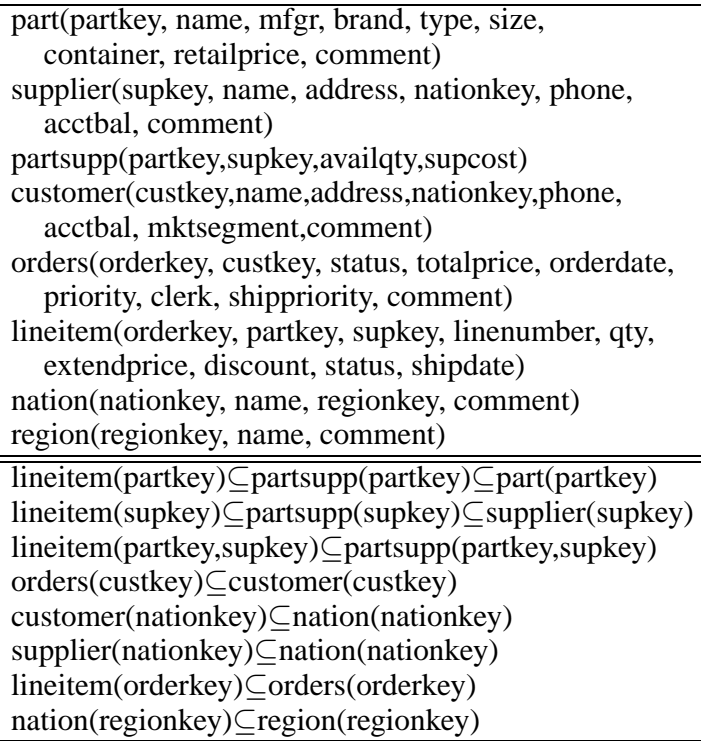

Figure 3: Abbreviated TPC-H Schema

Definition 2 A user query $Q=\left(N^{\prime}, E^{\prime}\right)$ on a join graph $J G=(N, E)$ is a subgraph of $J G$ such that $N^{\prime} \subseteq N$ and $E^{\prime} \subseteq E$.

For use in query inference, a user query reduces to a set of specifi ed nodes (relations) and edges (natural joins). A node is specifi ed if one or more of its attributes are required for a selection, projection, ordering, or grouping operation. A specifi ed edge is a natural join condition explicitly given by the user. The query interface must translate the user query into the required form.

Definition $3 A$ query interpretation $Q I=\left(N^{\prime}, E^{\prime}\right)$ on a join graph $J G=(N, E)$ is a connected subgraph of $J G$ such that $N^{\prime} \subseteq N$ and $E^{\prime} \subseteq E$.

A query interpretation is usually a tree, but may be a graph. We use the terms join tree to represent lossless joins and lossy join interpretation for query interpretations that include a lossy join. An ambiguous query has multiple join trees. A query may be unambiguous (single join tree) even if the schema contains ambiguity.

Definition 4 The query inference problem requires enumerating and ranking query interpretations of a query such that the query interpretation desired by the user is among the highest ranked interpretations.

Previously, query inference was defi ned as selecting a single interpretation for the user. This is impractical because a system will never be able to select the correct interpretation for every situation. We re-formulate the query inference problem as an information retrieval problem. The goal of query inference is to enumerate potential query interpretations

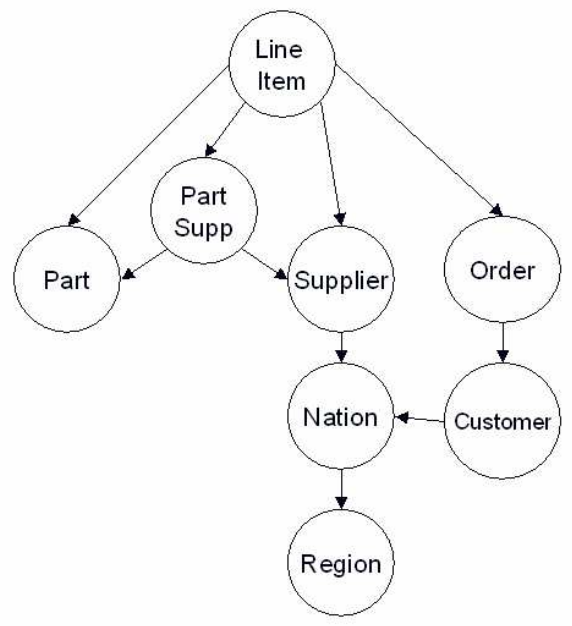

Figure 4: Join Graph for TPC-H Database

and present them to the user in order of the likelihood that the interpretation is what the user intends. This is similar to how a web search engine ranks pages according to the expected value for the user. Query inference then becomes two related problems. First, given a subset of nodes and edges of the join graph, the system must enumerate the possible connections between them. Then, it will rank the interpretations based on the specifi ed cost function.

\section{Precomputing Lossless Joins}

As the maximal sets of lossless joins depend solely on the ambiguity inherent in the schema and not on the input of a specific query, the maximal sets of lossless joins for the schema are precomputed. This moves the bulk of the computation from query time to a one time precomputation. If there is no ambiguity in a schema, then query inference is trivial with only one possible interpretation for any query. AutoJoin is designed to infer queries on schemas with ambiguity. There are two common sources of ambiguity in schemas:

- A single relation storing an entity that plays multiple roles results in a node in the join graph with two or more incoming edges.

- Multiple semantic relationships between entities result in directed cycles and strongly connected components in the join graph.

The first source of ambiguity is common as schemas have shared lookup tables used by multiple relations. An example in TPC-H is Nation that serves 
the two semantic roles of storing the nation of customers and the nation of suppliers. This form of ambiguity arises when distinct attributes have the same underlying domain of values. An example of the second source of ambiguity is a database storing employees and departments where an employee has a department and a department has a manager (which is also an employee). These two relationships result in a directed cycle between employee and department in the join graph. The number of query interpretations is dictated by the ambiguity inherent in the schema.

\subsection{Removing Shortcut Joins}

Semantically equivalent query interpretations with distinct sets of joins may exist in a database schema. It is critical that these equivalent joins be detected and only the core semantic join path preserved in the join graph, otherwise queries may be incorrectly determined to be ambiguous. A shortcut join is a join between two relations that is semantically equivalent to a longer join path of two or more edges. This is especially common in hierarchically structured databases where the primary key of one relation contains the primary key of its parent relation. We denote a natural join on foreign key attributes $X$ between two relations $R_{i}$ and $R_{j}$ as $R_{i} \bowtie_{X} R_{j}$. To simplify the discussion, the set of attributes $X$ is assumed to have the same name in both relations, although in practice this is not required.

Definition $5 A$ shortcut join between two relations $R_{i}$ and $R_{j}$ is a natural join on attributes $X$ where $X \subseteq$ $R_{i}$ and $X \subseteq R_{j}$ and there exists a join path $R_{i} \bowtie_{X_{1}}$ $T_{1} \bowtie_{X_{2}} T_{2} \bowtie_{X_{3}} \ldots \bowtie_{X_{n}} T_{n} \bowtie_{Z} R_{j}$ where $Z=X$ and $Z \subseteq X_{n} \subseteq X_{n-1} \ldots \subseteq X_{1}$.

A shortcut join is equivalent to the longer join path based on functional dependencies. The functional dependency $R_{i}[X] \rightarrow R_{j}$ of the shortcut join is equivalent to $R_{i}[Y] \rightarrow T[Z] \rightarrow R_{j}$ since $X \subseteq Y$ and $Z=X$. Shortcut joins can be detected while building the join graph. If a relation $R_{i}$ has two foreign keys on sets of attributes $Y$ and $X$ to relations $R_{j}$ and $R_{k}$ respectively, where $X \subset Y$ then the join from $R_{i}$ to $R_{k}$ on $X$ is a shortcut join and is not added to the join graph. Shortcut joins are maintained in a list and are re-inserted at query-time when the shortcut join can be used to reduce query execution times.

There are two shortcut joins in the TPC-H schema: LineItem to Part and LineItem to Supplier. By removing these joins, the number of maximal join trees in Figure 5 is reduced from 8 to 2 (join trees 1 and 5), and the number of unambiguous queries increases to $26 \%$ from $8 \%$ (Figure 8 ). At query-time, if a query specifi es only LineItem and Part, the path in the maximal join tree will be: LineItem-PartSupp-Part which

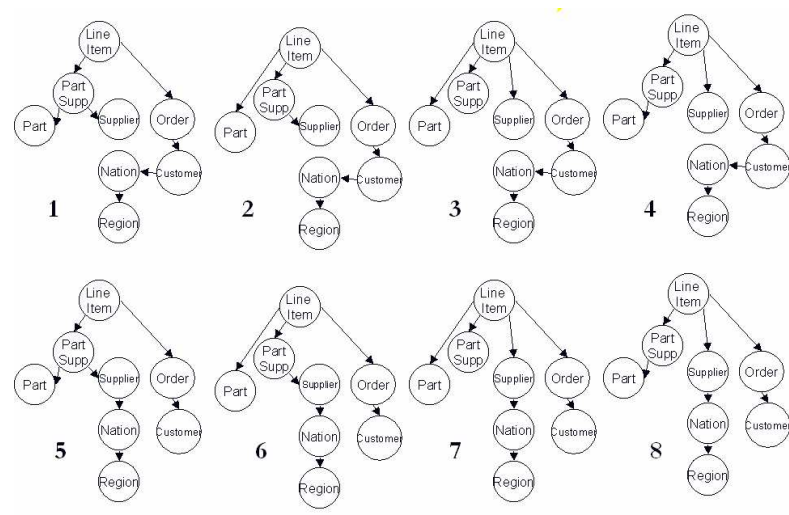

Figure 5: Maximal Join Trees for TPC-H Database

is replaced by the shortcut join LineItem-Part, as PartSupp is not required in the query.

\subsection{Generating Maximal Join Trees}

Previous approaches for generating maximal sets of lossless joins (join trees) expanded in all possible directions. The growing algorithm was ineffi cient, and often non-computable, for large graphs. Our approach constructs the sets of joins without expanding in all possible ways by growing maximal join trees only from identifi ed roots. Since lossless joins are trees, a root of a join tree cannot be a node with an incoming edge unless that node is in a strongly connected component (SCC). The algorithm identifi es nodes that have only outgoing edges or are in a strongly connected component with no incoming edges from outside the component. Only those nodes can be roots of maximal lossless joins. From each root, the reachable subgraph is found by traversing directed edges and an algorithm for calculating all spanning trees (Gabow and Myers, 1978) of the reachable graph is used to fi nd all maximal lossless joins for each subgraph. Repeating the process for every potential root produces all join sets without constructing any duplicates. The algorithm is in Figure 6.

As the number of spanning trees may be exponential (such as for a completely connected graph), the performance of the algorithm in the worst-case is exponential. In practice, database schemas do not exhibit the worst case and tend to have a reasonable number of maximal join trees. EMO outperforms a grow all ways approach both in time and number of edges and nodes visited. The performance improvement is because EMO does not duplicate effort and grows only from valid roots.

The following proofs demonstrate the correctness of the algorithm by showing that EMO generates all maximal join trees and only valid maximal join trees. 


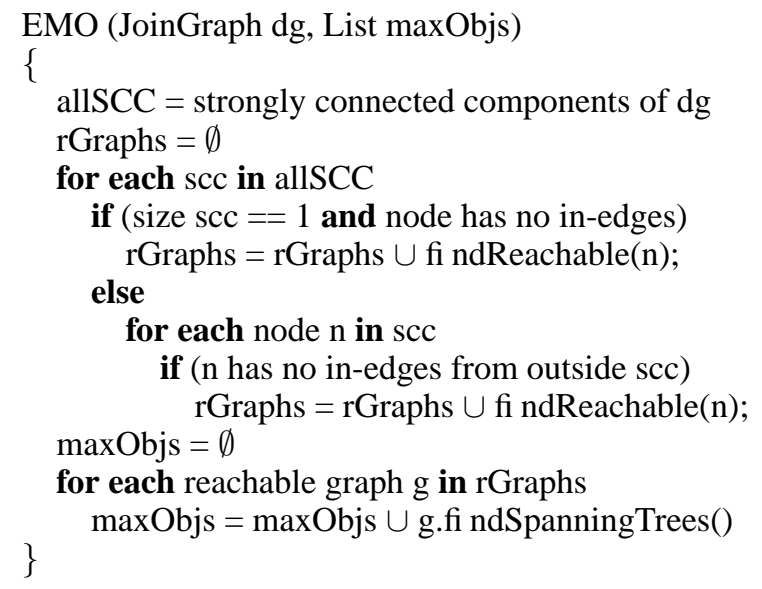

Figure 6: EMO Algorithm

Theorem 1 EMO produces all maximal join trees.

Proof: Proof by contradiction. Let $A$ be a maximal join tree that is not produced by EMO. Let $r$ be the root of $A$. Let $C$ be the strongly connected component containing $r$.

Case 1: If $C$ contains only one element, then $r$ must have no incoming edges. Otherwise, suppose it has incoming edge $(x, r)$. Adding $(x, r)$ to $A$ will produce a bigger tree containing $A$. That contradicts the fact that $A$ is a maximal join tree. So EMO will fi nd the reachable graph of $r$.

Case 2: If $C$ contains more than one element, then EMO fi nds the reachable graph of every node in $C$ with no incoming edges from outside $C$. If $r$ has no incoming edges from outside $C$, EMO will find the reachable graph of $r$. If $r$ has incoming edges from outside $C$, then similar to Case $1, A$ is not a maximal join tree.

In either case, $A$ is one of the spanning trees of the reachable graph of $r$. Thus, $A$ is produced by EMO.

Theorem 2 Any spanning tree produced by EMO is a unique maximal join tree.

Proof: Proof by contradiction. Suppose $A$ is a tree produced by EMO, but $A$ is not a maximal join tree. Then $A$ must be a subtree of some maximal join tree $B$. By Theorem 1, EMO produces $B$.

Case 1: Suppose $A$ and $B$ have the same root $r$. Then $A$ and $B$ are both spanning trees of the reachable graph of $r$. This contradicts the fact that $A$ is a smaller tree contained in $B$.

Case 2: Suppose the root $r A$ of $A$ is contained in $B$ but is not the root of $B$. Then $r A$ must have an incoming edge $e=(x, r A)$ where $x$ is not contained in $A$. EMO would not have found the reachable graph for $r A$ if it had an incoming edge unless $r A$ was in a strongly connected component $C$. Edge $e$ may be an incoming edge from outside $C$ or an edge within $C$. If it is an edge in $C, x$ must be in $A$ since $x$ would be reachable from $r A$. A contradiction as $x$ is not in $A$. If $e$ is an incoming edge from outside $C$, then EMO will not produce a spanning tree of the reachable graph from $r A$. Thus, $A$ would never be produced.

\section{Query-Time Processing}

It is critical to effi ciently translate a user query into one or more query interpretations for the relational database. This computation will occur for every query, where as the determination of maximal join trees is performed only once. The strategy involves quickly identifying and ranking all unique lossless interpretations of the user query, then if necessary generating the join possibilities with a lossy join. The algorithm for generating query interpretations executes the following steps:

- Identify the maximal join trees that contain all the user specifi ed nodes (and edges).

- Prune identifi ed trees to unique query interpretations, such that all leaf nodes are specifi ed nodes.

- If required, generate all join graphs with a single lossy join.

- Rank interpretations by the specifi ed cost function or default to minimal number of edges (joins).

To minimize processing time, several performance improvements are implemented. First, AutoJoin precomputes a reverse index where each relation (node) links to all the join trees containing this node. The intersection of the sets of join trees results in all of the unique join trees for the specifi ed nodes. Second, instead of recursively pruning leaf nodes of the maximal join tree, a minimal join tree is built by performing a union of the paths from the least common ancestor of all requested nodes to each requested node. The ancestor lists are precomputed with the maximal join trees prior to query time.

\subsection{Extension to Lossy Joins}

For queries that do not have a lossless interpretation or require additional interpretations, query interpretations involving a lossy join are generated. These additional interpretations are created by the union of pairs of maximal join trees that together contain all the requested nodes and have at least one node in common. The union of these join trees will contain one or more nodes with more than one incoming edge. A prune method generates all minimal interpretations with a single lossy join. The AutoJoin inference engine generates query interpretations as a connected graph con- 
taining all of the specifi ed nodes with a single node having two incoming edges.

\subsection{Beyond Natural Joins}

The main focus of this work is on natural joins of foreign keys as they are the most common type of join. However, query inference is not restricted to queries with only natural joins. In general, any complex join condition can be specifi ed and the query inference engine will infer any natural joins still required to complete the query. A theta join is handled by merging the two join nodes in the original join graph and updating the edges. Due to EMO's effi ciency, it is possible to compute the maximal join trees for the modifi ed join graph and apply the regular query inference algorithm. Maier and Ullman provide a method for inferring queries involving tuple variables (Maier and Ullman, 1983). Tuple variables allow one or more relations to occur in a query multiple times, allowing for query inference of more complicated queries. Any query interface built on the AutoJoin inference engine may allow the user to specify such complex queries by specifying additional nodes and edges required to be in the query interpretations. Query inference can be applied to any query including ones that use subqueries, complex join predicates and outer joins.

\section{Performance Experiments}

The AutoJoin inference engine is implemented in Java and uses the JDBC API to extract schema information. All schemas are in production use at various organizations or extracted from the Internet. The largest schema is $c a B I O$ from the NCI Cancer Grid project (caBIG) ${ }^{2}$ which contains 149 nodes, 213 edges, and 1253 maximal join trees. The experiments were performed on a $1.3 \mathrm{GHz}$ AMD Athlon with 512 $\mathrm{MB}$ of memory running Windows XP.

The fi rst experiment compares the performance of EMO with the grow all ways approach used in (Maier and Ullman, 1983; Semmel and Mayfi eld, 1997; Hristidis and Papakonstantinou, 2002). The results in Figure 7 show that EMO signifi cantly outperforms the grow all ways approach. The grow all ways approach is very inefficient and cannot complete the $c a B I O$ schema without running out of memory. ${ }^{3}$ Both approaches are comparable for very small graphs. EMO outperforms on large graphs by an order of magnitude, which is especially important when schema evo-

\footnotetext{
${ }^{2}$ http://cabig.nci.nih.gov/

${ }^{3}$ The grow all ways algorithm is either CPU or memory constrained depending on breadth first or depth first growth. The DFS approach did not complete for the Claims or $C a B I O$ database.
}

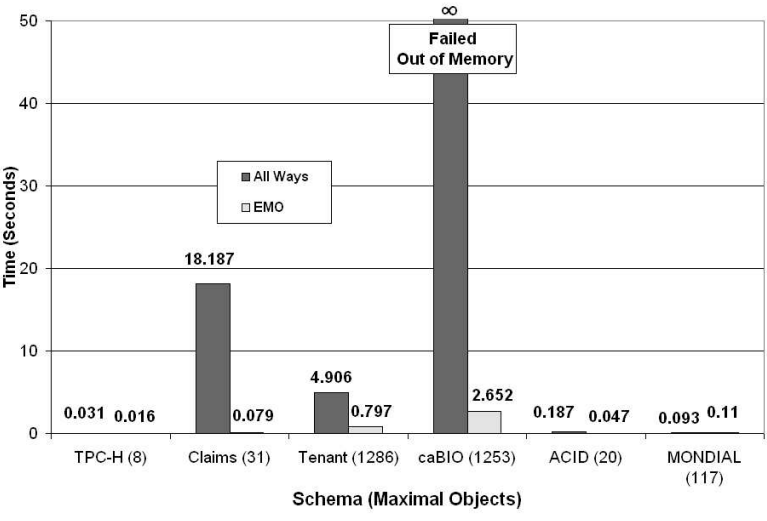

Figure 7: Time to Compute Maximal Join Trees

lution is frequent. EMO is able to handle schemas that could not even be processed by the existing approaches. EMO's absolute performance is suffi ciently fast to support generation of maximal join trees at query-time for almost all database schemas.

The second experiment determines how removing shortcut joins reduces ambiguity. We use the TPC-H schema and determine the percentage of queries that are unambiguous before and after removing shortcut joins. All 255 potential queries were treated as equally likely (all queries of one table, of two tables, etc.). Removing shortcut joins improved the number of unambiguous queries from $8 \%$ to $26 \%$ (see Figure 8). Of the 22 benchmark TPC-H queries, removing shortcut joins made $68 \%$ of the queries unambiguous versus $45 \%$ originally. Several benchmark queries contain nested subqueries. We consider a query to be unambiguous if both the join tree for the subquery and outer query is unambiguous. Query 5 contains a lossy join, and two queries (7 and 8) require two copies of Nation that must be specifi ed by the user. The hierarchical EDS schema shows an even greater improvement by removing shortcut joins.

The overhead of performing query inference for each query must be minimal. In a third experiment, we determine the time to perform query inference on various schemas. We calculated the average time to infer the joins for the 22 benchmark TPC-H queries, for all possible 255 TPC-H queries, and for two table lossless queries in the other sample databases. As Figure 9 shows, the average query inference time is well below $10 \mathrm{~ms}$ even for large schemas. Removing shortcut joins improves the time for TPC-H. The inference time for $\mathrm{CaBIO}$ only slightly increases despite the signifi cantly larger schema size. By building query interpretations using least common ancestor versus recursive pruning, the average inference time decreased from $126 \mathrm{~ms}$ to $2.7 \mathrm{~ms}$ on caBIO. The lossy join in 


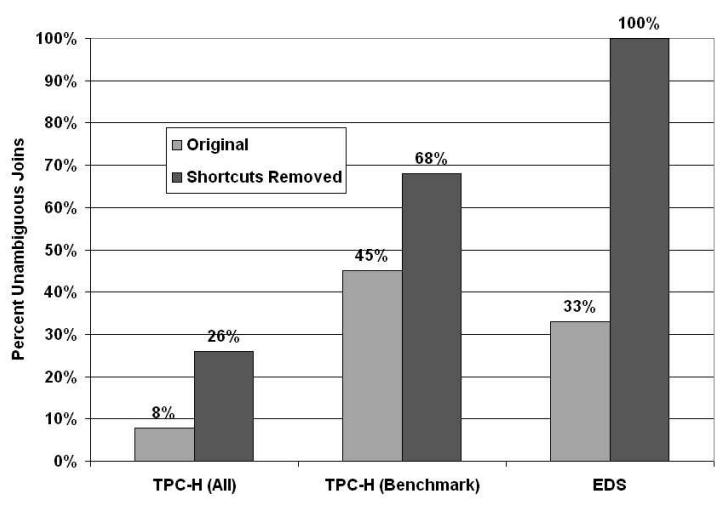

Figure 8: Reducing Ambiguity by Removing Shortcut Joins

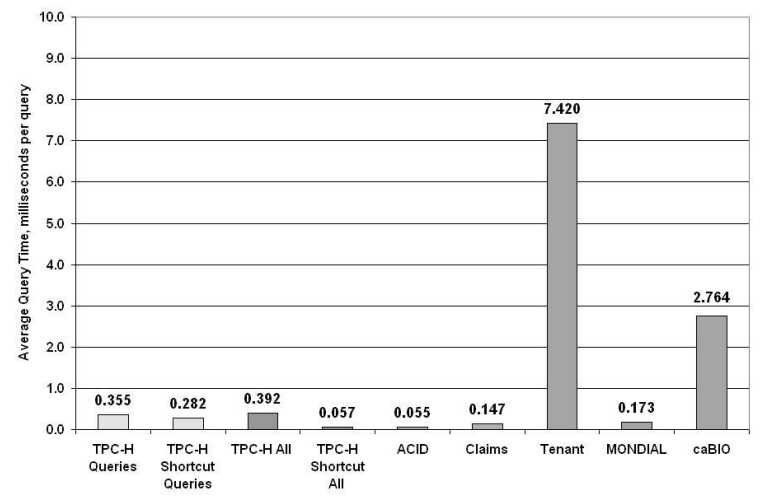

Figure 9: Query Inference Time

TPC-H query 5 is specifi ed for this experiment. If it was not specifi ed, the time to compute all lossless and one-lossy interpretations (using the strategy in Section 5.2) was $16 \mathrm{~ms}$ (after removing shortcut joins). Thus, the time to infer a query is minimal.

\section{Conclusion}

By combining the algorithms to address the challenges of query inference into a single inference engine, AutoJoin provides a comprehensive tool for developing the next generation of query interfaces. In addition, it provides a method to create SQL queries without requiring full knowledge of the database. Inference is possible on larger and more complex schemas, due to the effi cient maximal join tree algorithm EMO. By precomputing these potential joins, the majority of computation occurs only once, prior to query time. Identifi cation and removal of semantically equivalent shortcut joins reduces the number of ambiguous queries further improving inference. Predetermined look up tables combined with indexing of potential joins allows for effi cient identifi cation of joins for each query. Numerous performance experiments on diverse schemas demonstrate that the time to infer these joins for each query is minimal. In addition, the complexity of queries inferred exceeds any previous individual inference method. AutoJoin is a general inference engine confi gurable to specifi c query interface requirements. This allows for the continued development of simpler keyword, conceptual, and natural language query interfaces without the burden of developing a scalable and efficient join determination algorithm. Overall, query inference is a practical query tool to incorporate into query languages and database systems.

\section{REFERENCES}

Agrawal, S., Chaudhuri, S., and Das, G. (2002). DBXplorer:A System for Keyword-Based Search Over Relational Databases. In IEEE ICDE, pages 5-16.

Balmin, A., Hristidis, V., and Papakonstantinou, Y. (2004). ObjectRank:Authority-Based Keyword Search in Databases. In $V L D B$, pages 564-575.

Catarci, T. (2000). What happend when Database Researchers met Usability. Information Systems, 25(3):177-212.

Gabow, H. and Myers, E. (1978). Finding All Spanning Trees of Directed and Undirected Graphs. SIAM Journal of Computing, 7(3):280-287.

Hristidis, V. and Papakonstantinou, Y. (2002). DISCOVER:Keyword Search in Relational Datbases. In $V L D B$, pages 670-681.

Maier, D. and Ullman, J. (1983). Maximal Objects and the Semantics of Universal Relation Databases. TODS, 8(1):1-14.

Owei, V. and Navathe, S. (2001). Enriching the conceptual basis for query formulation through relationship semantics in databases. Information Systems, 26(6):445-475.

Popescu, A., Etzioni, O., and Kautz, H. (2003). Towards a theory of natural language interfaces to databases. In IUI.

Semmel, R. and Mayfield, J. (1997). Automated Query Formulation using an Entity-Relationship Conceptual Schema. Intelligent Information Systems, 8:267-290.

Wald, J. and Sorenson, P. (1984). Resolving the Query Inference Problem Using Steiner Trees. TODS, 9(3):348-368.

Zhang, G., Meng, F., Kong, G., and Chu, W. (1999). Query Formulation from High-level Concepts for Databases. In User Interfaces to Data Intensive Systems. 